\title{
Anaerobic Biotransformation and Mobility of Pu and of Pu-EDTA
}

(Final report)

\author{
Luying Xun ${ }^{1}$ and Harvey Bolton Jr. ${ }^{2}$, \\ ${ }^{1}$ Washington State University, Pullman, WA, and ${ }^{2}$ Pacific Northwest National Laboratory, \\ Richland, WA
}

\begin{abstract}
The enhanced mobility of radionuclides by co-disposed chelating agent, ethylenediaminetetraacetate (EDTA), is likely to occur only under anaerobic conditions. Our extensive effort to enrich and isolate anaerobic EDTA-degrading bacteria has failed. Others has tried and also failed. To explain the lack of anaerobic biodegradation of EDTA, we proposed that EDTA has to be transported into the cells for metabolism. A failure of uptake may contribute to the lack of EDTA degradation under anaerobic conditions. We demonstrated that an aerobic EDTA-degrading bacterium strain BNC1 uses an ABC-type transporter system to uptake EDTA. The system has a periplasmic binding protein that bind EDTA and then interacts with membrane proteins to transport EDTA into the cell at the expense of ATP. The bind protein EppA binds only free EDTA with a $K_{d}$ of $25 \mathrm{nM}$. The low $K_{d}$ value indicates high affinity. However, the $K_{d}$ value of Ni-EDTA is $2.4 \times 10^{-10} \mathrm{nM}$, indicating much stronger stability. Since Ni and other trace metals are essential for anaerobic respiration, we conclude that the added EDTA sequestrates all trace metals and making anaerobic respiration impossible. Thus, the data explain the lack of anaerobic enrichment cultures for EDTA degradation. Although we did not obtain an EDTA degrading culture under anaerobic conditions, our finding may promote the use of certain metals that forms more stable metal-EDTA complexes than $\mathrm{Pu}(\mathrm{III})$-EDTA to prevent the enhanced mobility. Further, our data explain why EDTA is the most dominant organic pollutant in surface waters, due to the lack of degradation of certain metal-EDTA complexes.
\end{abstract}

Introduction. The complexation of radionuclides (e.g., plutonium $(\mathrm{Pu})$ and $\left.{ }^{60} \mathrm{Co}\right)$ by codisposed ethylenediaminetetraacetate (EDTA) has enhanced their transport in sediments at DOE sites. Our previous NABIR research investigated the aerobic biodegradation and biogeochemistry of Pu(IV)-EDTA. Plutonium(IV) forms stable complexes with EDTA under aerobic conditions and an aerobic EDTA degrading bacterium can degrade EDTA in the presence of Pu and decrease Pu mobility. However, our recent studies indicate that while $\mathrm{Pu}(\mathrm{IV})$-EDTA is stable in simple aqueous systems, it is not stable in the presence of relatively soluble Fe(III) compounds. Formation of Fe(III)-EDTA releases Pu(IV). Since most DOE sites have Fe(III) containing sediments, $\mathrm{Pu}(\mathrm{IV})$ can not be the mobile form of Pu-EDTA in groundwater. The only other Pu-EDTA complex stable in groundwater relevant to DOE sites would be Pu(III)-EDTA, which only forms under anaerobic conditions. The project has three related tasks. Task 1 is to investigate the biogeochemistry of Pu-EDTA under anaerobic/reducing conditions. Task 2 is to study microbial reduction of $\mathrm{PuO} 2$ and $\mathrm{Pu}(\mathrm{IV})-$ EDTA. Task 3 is to enrich and isolate anaerobic EDTA degrading bacteria. The report is focused on Task 3, which was conducted at Washington State University. Tasks 1 and 2 were done at Pacific Northwest National Lab, and a separate final report will be submitted. 
Isolation of a new EDTA degrader. We have previously studied EDTA and nitrilotriacetate (NTA) by aerobic bacteria and worked out the complete metabolic pathway of EDTA and nitrilotriacetate (NTA), identified and characterized the genes and enzymes involved (refs). Interestingly, same genes and enzymes are responsible for the degradation of both EDTA and NTA in aerobic bacteria. However, the current project is focused on enrich and isolate anaerobic EDTA degraders. We have tried to enrich anaerobic bacteria that degrade NTA and EDTA. We observed algal growth in one of the anaerobic NTA-degrading enrichments originated from the anaerobic sludge of Pullman Wastewater Treatment Plant (Pullman, WA). Since NTA was the only nitrogen source, we have hypothesized that a bacterium used NTA and released ammonium for the alga to grow. The hypothesis was confirmed because the alga enrichment failed to grow in the mineral medium when NTA was omitted, and the isolated alga did not use NTA as a nitrogen source, either. Further, several NTA-degrading bacteria were isolated from the enrichment. After repeated transfer of the alga enrichment into a mineral medium containing only EDTA as nitrogen source, an alga enrichment with EDTA as the nitrogen source was obtained. EDTA can be replaced with NTA or nitrate as the nitrogen source. From the enrichment, an EDTA-degrader was isolated. Thus, the enrichment contained bacteria that degrade EDTA and released ammonia from EDTA for the alga to grow. The 16S rRNA gene sequence (Appendix) showed 99.4\% match with Agrobacterium tumefaciens, and 93\% match with the known EDTA-degrading bacterium Mesorhizobium sp. BNC1. The bacterium use both EDTA and NTA as the sole carbon and energy source under aerobic condition, and it did not use EDTA or NTA under anaerobic conditions. Although the enrichment culture was under anaerobic conditions, alga produced $\mathrm{O}_{2}$ for the bacterium to degrade EDTA and release ammonium. Repeated attempts failed.

Understanding why EDTA is recalcitrant in nature. We have previously characterized the metabolic pathway of EDTA and NTA metabolism by aerobic bacteria. However, we did not characterize how EDTA is transported into cells for metabolism. Here, we further characterize EDTA uptake by bacteria. We found that only free EDTA is transported into cells for degradation. In the environment, EDTA forms stable complexes with some transitional metals. Since the stable complexes are not transported into bacterial cells, they become recalcitrant to biodegradation. Consequently, EDTA is now the most abundant pollutant in natural waters. Our results have been published in "Journal of Bacteriology". We also gave an invited presentation on the biochemistry of EDTA uptake and metabolism at "Complexing agents between science, industry, authorities and users” in Ascona, Switzerland in March 2007. In addition, we have characterized the structure and function of an enzyme (EmoB) involved in EDTA degradation and the results were published in "Journal of Biological Chemistry".

\section{Possible explanations on why EDTA is not degraded under anaerobic conditions.}

Anaerobic enrichment culture with EDTA as the sole carbon source and nitrate as the terminal electron acceptor for anaerobic respiration has resulted in enrichments that produce ammonia. However, a stable enrichment that consumes EDTA has not been obtained. Enrichments with different sediments of lakes, rivers, and oceans were tested in different mineral media for enrichments. Although initial growth was frequently observed, stable enrichment cultures were never obtained. Because anaerobic degradation of EDTA has never been reported, we conclude that anaerobic EDTA degraders are hard to enrich. On the basis of our current understanding of EDTA chelating properties and the uptake of free EDTA (not metal-EDTA complexes) into bacterial cells for metabolism, we believe that EDTA form stable complexes with trace metals, 
such as nickel, cobalt and cupper, making them unavailable to bacteria. It is known that bacteria require these metals under anaerobic conditions as enzyme cofactors, e.g. [NiFe] hydrogenases and other proteins involved in anaerobic electron transport systems. Thus, trace metals are often added to culture media for anaerobic bacteria. EDTA has high affinities to the trace metals, making them unavailable to anaerobic bacteria. Therefore, we believe that EDTA is not likely to be degraded under anaerobic conditions due to EDTA's chelation and sequestration of trace metals, which are essential for the growth of most anaerobic bacteria.

Environmental consequences of EDTA recalcitrance under anaerobic conditions. The Department of Energy (DOE) has Pu- and EDTA-contaminated sediments at several sites (6). The co-contamination was a result of extensive use of EDTA to decontaminate nuclear materials and to process nuclear wastes (4). Since EDTA was stable under anaerobic conditions. It is expected that it can enhance the transport of radionuclides through groundwater. This expectation is in agreement with numerous reports. The groundwater transport of $\mathrm{Pu}$ and ${ }^{60} \mathrm{Co}$ was enhanced by co-disposed EDTA at Oak Ridge National Laboratory, Tennessee (5); Chalk River Nuclear Laboratories, Canada (3), Maxey Flats, Kentucky (1), and the Hanford Site, Washington (2). Thus, bioremediation may not be a valid approach for EDTA removal under anaerobic conditions. Alternative approaches may be used, e.g. the use of certain metals that forms more stable metal-EDTA complexes than Pu(III)-EDTA to prevent the enhanced mobility.

\section{Meeting presentation:}

Xun, L., and H. Bolton, Jr. 2007. Biochemistry of EDTA uptake and metabolism by EDTAdegrading bacterium BNC1. Invited presentation at the "Complexing agents between science, industry, authorities and users”, Ascona, Switzerland. (Expenses were paid by the meeting).

\section{Publications:}

Zhang, H., J. P. Herman, H. Bolton, Jr., Z. Zhang, S. Clark, and L. Xun. 2007. Evidence indicating that a bacterial ABC-type transporter imports free EDTA for metabolism. J. Bacteriol. 189:7991-7997.

Nissen, M. S., B. Youn, B. D. Knowles, J. W. Ballinger, S.-Y. Jun, S. M. Belchik, L. Xun, and C. Kang. 2008. Crystal structures of NADH:FMN oxidoreductase (EmoB) at different stages of catalysis. J. Biol. Chem. 283:28710-28720.

\section{Reference:}

1. Cleveland, J. M., and T. F. Rees. 1981. Characterization of plutonium in Maxey Flats radioactive trench leachates. Science 212:1506-1509.

2. Freeman-Pollard, J. R., J. A. Caggiano, and S. J. Trent. 1994. Engineering evaluation of the GAO-RCED-89-157, Tank 241-T-106 vadose zone investigation. BHI-00061. Bechtel Hanford, Inc.

3. Killey, R. W. D., J. O. McHugh, D. R. Champ, E. L. Cooper, and J. L. Young. 1984. Subsurface cobalt-60 migration from a low level waste disposal site. Environ. Sci Technol 18:148-157. 
4. McFadden, K. M. 1980. Organic components of nuclear wastes and their potential for altering radionuclide distribution when released to soil. PNL-2563. National Technical Information Service, Springfield, VA.

5. Means, J. L., D. A. Crerar, and J. O. Duguid. 1978. Migration of radioactive wastes: Radionuclide mobilization by complexing agents. Science 200:1477-1486.

6. Riley, R. G., and J. M. Zachara. 1992. Chemical Contaminants on DOE Lands and Selection of Contaminant Mixtures for Subsurface Science Research. DOE/ER-0547T. National Technical Information Service, U.S. Department of Commerce, Springfield, VA. 